\title{
A novel compact CPW band-stop filter using O-DGS configuration
}

\author{
Elmahjouby Sghir ${ }^{1}$, Ahmed Errkik ${ }^{2}$, Jamal Zbitou ${ }^{3}$, Larbi El abdellaoui ${ }^{4}$, \\ Abdelali Tajmouati ${ }^{5}$, Mohamed Latrach ${ }^{6}$ \\ 1,2,3,4,5 LMEET Laboratory FST of Settat Hassan 1st University, Morocco \\ ${ }^{6}$ Microwave group ESEO Angers France, France
}

\section{Article Info}

Article history:

Received Jan 6, 2019

Revised Jun 25, 2019

Accepted Jul 5, 2019

\section{Keywords:}

Coplanar line

Defected ground structure

(DGS)

Multi-stop stopband

Stopband filter

\begin{abstract}
A novel and compact multi-band stop filter using two different structures of circular DGS and circular DGS loaded with O-DGS unit, has good selectivity and wide upper pass band performance with good rejection based on defected ground structure (DGS) integrating a circular ring resonator to suppress undesired spurious bands frequency or harmonics in radio frequency and microwave circuits is proposed. An equivalent circuit model has been derived for the both structures of circular DGS and circular DGS loaded. The presented filter has a compact size because the circular ring resonator is integrated in DGS, the both of them are etching in the ground plane. The proposed filter presents an optimization method for generating multi-band characteristics without using a periodic cells or other complicate topology. The simulated results by ADS Agilent are presented and compared with the results simulated by two others software: CST and HFSS. Good agreement between the three simulated results is observed.
\end{abstract}

Copyright (c) 2019 Institute of Advanced Engineering and Science. All rights reserved.

\section{Corresponding Author:}

Elmahjouby Sghir,

Department of Physics,

University of Hassan $1^{\text {st }}$ Morocco.

Email: elmahjouby.sghir@gmail.com

\section{INTRODUCTION}

Coplanar transmission line offers an attractive solution to compact and ease low cost design of microwave circuits for modern wireless applications systems due to their many advantages as lightweight and low volume. Also, the planar geometry of ground which can be etched to create different effect on the frequency response. EBG 'Electromagnetic Band Gap' and DGS 'Defected ground structures' are two of these technical structures.

DGS is an etching in the ground plane of the planar circuits, which permits to change the response of the microwave circuit and obtained some new transmission characteristics of the component [1-6]. However, DGS structure have been employed in a lot off microwave circuits like filters [2], power dividers/combiners [3], and microstrip antenna [4], for improving the performance of microwave circuits and to have a small seize of structures.

Defected ground structures (DGS) has been obtained from the photonic bandgap structure idea (PBGs) [5]. This later was utilized firstly at optical field, after it has been derived into planar circuits in electromagnetic microwave frequency with the name of electromagnetic bandgap (EBG) [6]. Although EBG structures have difficulty in its modeling and it is one of their basic disadvantages. So DGS structure gives a response of this problem and simplified the modeling of the RLC-equivalent circuits. There are many effective approaches available to implement filters with dual stopbands response, one of them is the Periodic structures by cascading the unit DGS structures in the metal layer. Nevertheless, this filter has a relatively large size (because numbers of units added). Here in this paper, a solution to that is to use the loaded DGS 
structure to improve the response performance of filter, without adding periodic structures, and therefore retains a compact size.

In this article, using new DGS loaded structures are being exploited to reach maximum miniaturization and minimum losses, and give a good solution to improve stopband and passband filters. The extracted parameters of the both proposed structures can be interpreted based on the circuit analysis theory.

\section{RESEARCH METHODCOPARISON OF VARIOUS DGS APPLIQUED IN MICROWAVE FILTERS}

Nowadays DGS is widely used in microwave technologies, when filters is one of these devices, especially with coplanar waveguide technology, because the planar geometry of the ground offers a sufficient area to etched any geometrical defects. Figure 1 shown the various DGS geometrical pattern.

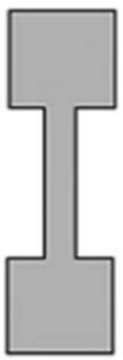

(a)

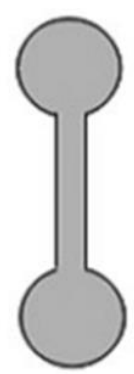

(b)

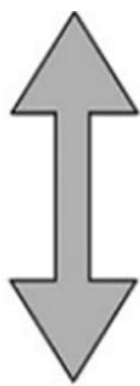

(c)

Figure 1. Various DGS geometrical pattern: (a) triangular shaped (b) square shaped (c) circular shaped

Deferent DGS shapes of geometries on the ground plane of cpw line have been reported in the literature as triangular shaped [7-9], square shaped [10,11] and in this paper a circular shaped is discussed with the technique of loaded DGS. There are two major approaches available for utilizing the performance of DGS, one is DGS unit, which permits to change the response of the microwave circuit and obtained some new transmission characteristics of the component depend of the DGS geometrical pattern [1-6]. The others periodic DGS, as [9], which a three cells periodical triangle DGSs was designed in an attempt to improve the performance of LPF and improved stop band and suppression performance. However, in this work a novel aspect to improve and implementation of a multi-band filter, with utilizing the loaded DGS structure.

\section{BASIC DGS UNIT OF THE COMPACT FILTER}

The building structure of the proposed stop band filter is a circular DGS resonator etched in the ground plane of coplanar waveguide cpw line that is directly connected to the line gap with the two other slots. A schematic of the proposed stopband filter with DGS circular structure is shown in Figure 2. It consists of a $50 \Omega$ conventional coplanar transmission line, with a signal line width of $\mathrm{W}=1.5 \mathrm{~mm}$ and the gap width, $\mathrm{G}=0.204 \mathrm{~mm}$. The proposed DGS stopband filter is designed on a FR4 substrate with a relative dielectric constant 4.4 and a thickness of $1.6 \mathrm{~mm}$.

Figure 2 shows, the DGS structure here is a circular slot etched in the ground plane with $\mathrm{r} 1=2 \mathrm{~mm}$. This circular DGS is connected to the line gap by a rectangle transverse slot with length of ls $(1 \mathrm{~s}=2.5 \mathrm{~mm})$ and width of ws (ws $=0.204 \mathrm{~mm}$ ). The proposed circuit is optimized by using Momentum based on moments method.

The complete analysis of the filter done with EM simulation tool. Figure 3 displays the insertion loss (S21) and return loss (S11) of DGS circular structure. A DGS is constructed by etching a defected circular on the ground plane and have been employed for changing the effective capacitance and inductance of a coplanar line formed on the circuit, as a result shown in Figure 3, a DGS improving the performance of stopband filter, so it plays the role of resonator. 


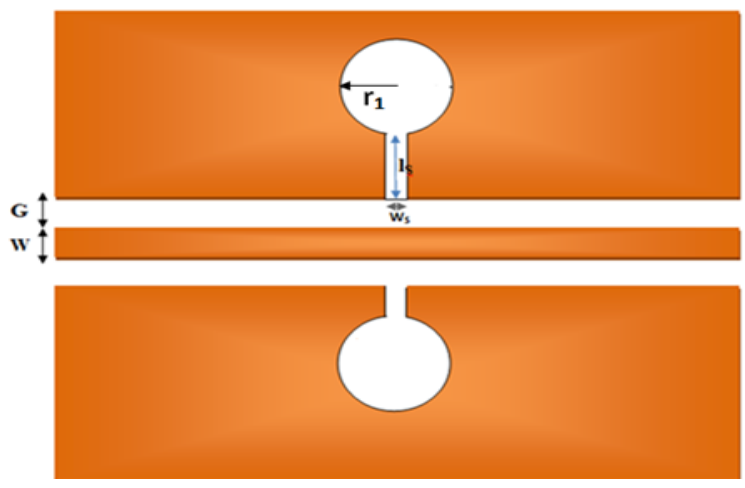

Figure 2. CPW line with Circular DGS structure

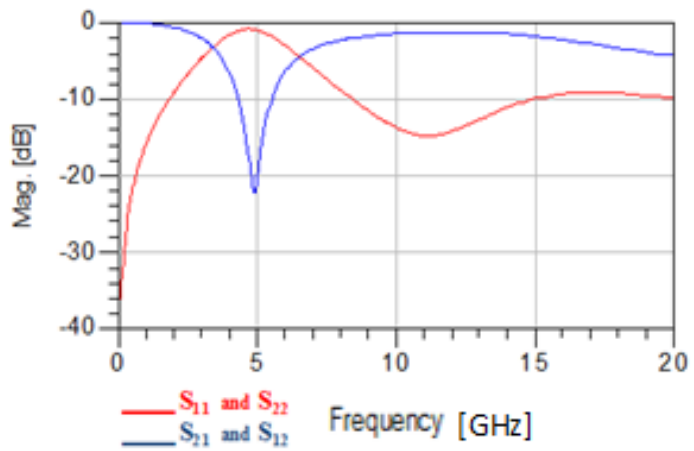

Figure 3. Simulated S-parameters versus frequency for the CPW DGS Circular structure of BandStop

As noted from the S-parameter studies, we have a stopband behavior of the circuit with a good bandstop around $5 \mathrm{GHz}$. And insertion loss (S21) is better than $-10 \mathrm{~dB}$ and a low return loss (S11) in the stopband. Also high out-band passing from $7 \mathrm{GHz}$ to $15 \mathrm{GHz}$ and return loss (S11) below $-10 \mathrm{~dB}$ in the passband without any others harmonics response. Here, the resonant frequency dependents by circular etched parameters and size of the slot.

The results of EM simulation and equivalent circuit are all shown in Figure 4. An inductive capacitive and resistance (RLC) equivalent circuit can be used to model the used DGS. As the Figure 4 shows, there is a good agreement between the EM and circuit simulated S-parameters. This good agreement demonstrates the validity of equivalent circuit model for the proposed circular DGS filter. Its RLC-equivalent circuit of the DGS cell is proposed, and the different parameters can be extracted. The $\mathrm{R}, \mathrm{L}$ and $\mathrm{C}$ resonant circuit is shown in Figure 5. The resistance $\mathrm{R}$ is added to the LC circuit to model the radiation, conductor and dielectric losses [12]. The different parameters can be extracted as follows [13].

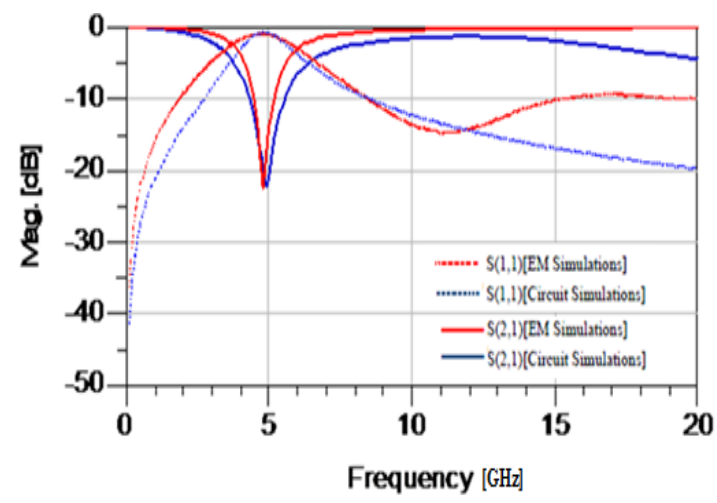

Figure 4. EM and circuit simulated S-parameters of CPW DGS circular structure of band stop 


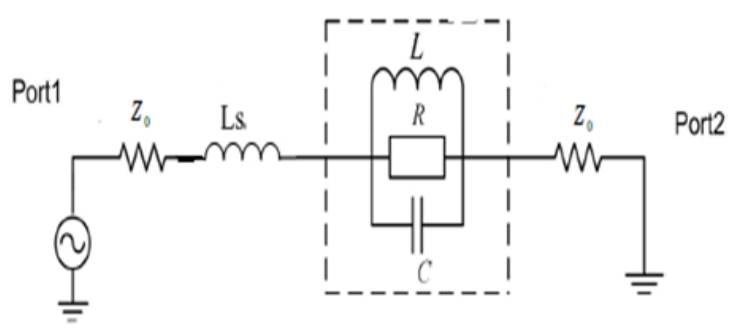

Figure 5. RLC-equivalent circuit for the CPW DGS filter

$$
\begin{aligned}
& \mathrm{C}=\frac{\mathrm{w}_{\mathrm{c}}}{2 \mathrm{Z}_{0}\left(\mathrm{w}_{0}^{2}-\mathrm{w}_{\mathrm{C}}^{2}\right)} \\
& \mathrm{L}=\frac{1}{4\left(\pi \mathrm{f}_{0}\right)^{2} \mathrm{C}} \\
& \mathrm{R}=\frac{2 \mathrm{Z}_{0}}{\sqrt{\frac{1}{\left|\mathrm{~S}_{11}\left(\mathrm{w}_{0}\right)\right|^{2}}-\left(2 \mathrm{Z}_{0}\left(\mathrm{w}_{0} \mathrm{C}-\frac{1}{\mathrm{w}_{0} \mathrm{~L}}\right)\right)^{2}-1}}
\end{aligned}
$$

where, Z0 is the characteristic impedance of the coplanar wave guide line, w0 is the angular resonance frequency, wc is the $-3 \mathrm{~dB}$ cutoff angular frequency, and $\mathrm{S} 11(\mathrm{w})$ is the input reflection coefficient of the equivalent circuit, which can be obtained from EM simulation results [14].

Table 1. Parameters of proposed RLC-Equivalent circuit for CPW DGS filter

\begin{tabular}{cc}
\hline Parameter & Value \\
\hline $\mathrm{f} 01$ & $5 \mathrm{GHz}$ \\
$\mathrm{R} 1$ & $1229.8 \Omega$ \\
$\mathrm{C} 1$ & $0.8137 \mathrm{pF}$ \\
$\mathrm{L} 1$ & $1.352 \mathrm{nH}$ \\
\hline
\end{tabular}

\section{THE PROPOSED DEVICE OF CIRCULAR DGS LOADED}

This new loaded circular DGS is proposed to widen the pass band of this filter after the reject band, without changing the center frequency of band stop, we propose loading circular DGS etched by a circular resonator which is composed of two resonators, as shown in Figure 6.

The suggested filter consists of two resonators, when the initial DGS structure stopband filter design is modified and loaded by introducing a circular resonator. The new structure with O-configuration used in this filter includes multiband response, passband component consisting of the new effective capacitance and inductance response of coplanar line.

The parameters of the first DGS stopband filter are kept unchanged while dimensions of the new circular resonator are introduced, as shown in Figure 6. The radius of new circular resonator is $\mathrm{r} 2=1.95 \mathrm{~mm}$. Figure 7 shows the simulated S-parameters of the proposed device of DGS loaded.

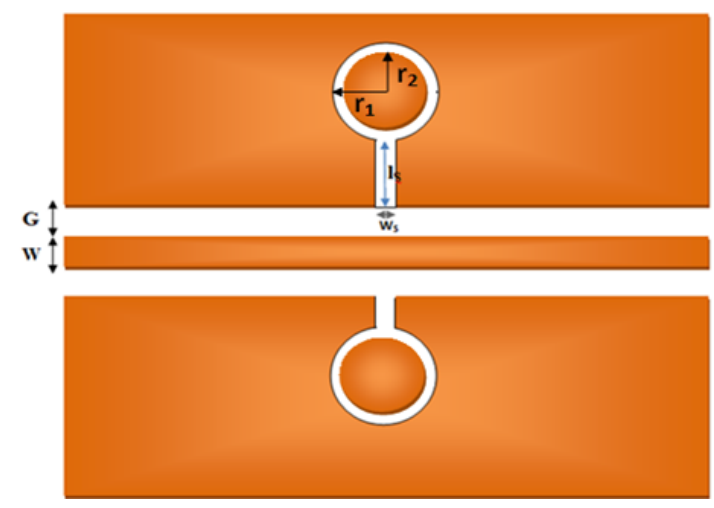

Figure 6. The proposed device of DGS Loaded for stopband filter 


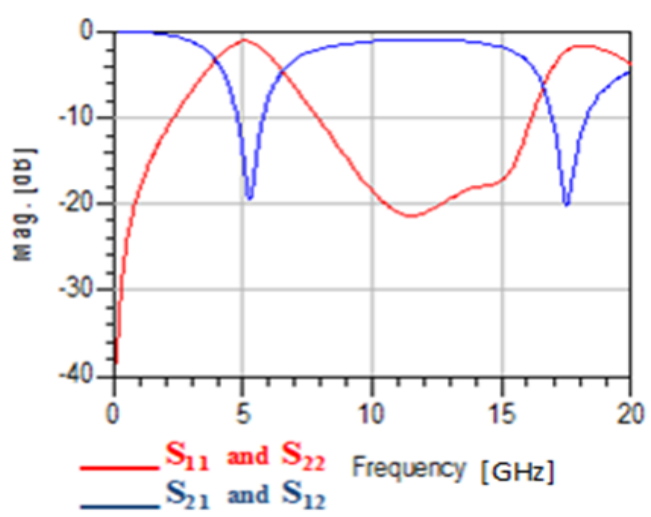

Figure 7. Simulated S-parameter of the proposed DGS loaded device

As discussed in the previous section, the circuit above study is composed of two resonators, the first O-DGS and the introduced circular unit cell resonators, as shown in Figure 6. The reason for this improvement lies in the EM simulation results of the loaded DGS structure, which is present two, reject bands spearing with a large passband, as shown in Figure 7. According to final dimensions of structure, the simulation result is given by HFSS and CST Microwave Studio in order to compare the results. The Figure 8 shows the compared s-parameters of the three simulations.

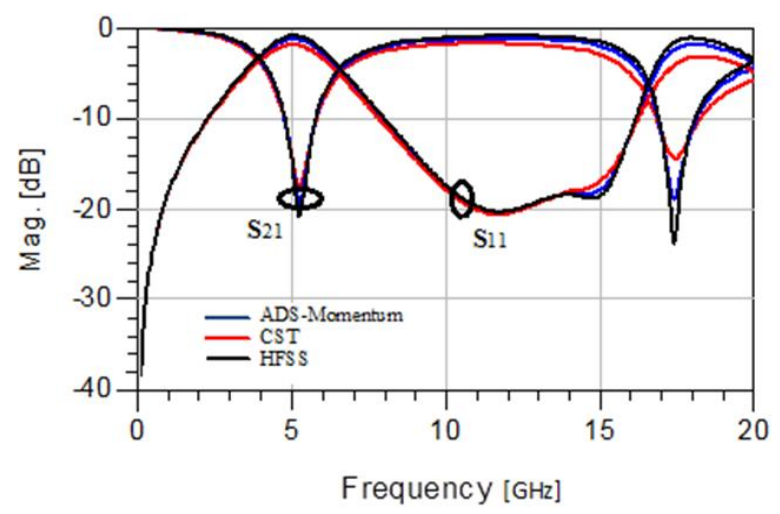

Figure 8. Comparison between different methods of simulation

As a result, the new circuit with DGS loaded include two stopped bands intermediate wide passband. The first stopband resonance is always around $5 \mathrm{GHz}$, as in first unloaded DGS structure, it is approximately centered between $3.5 \mathrm{GHz}$ and $6.5 \mathrm{GHz}$, and it exhibits more than $10 \mathrm{~dB}$ stopband with minimum $|\mathrm{S} 21|$ of $-20 \mathrm{~dB}$ around $5 \mathrm{GHz}$. The second reject band is around $17.5 \mathrm{GHz}$ with insertion loss is around $20 \mathrm{~dB}$. Also we can notice that the $20 \mathrm{~dB}$ pass-band is from $6.5 \mathrm{GHz}$ to $16.1 \mathrm{GHz}$, with insertion loss is less than $2 \mathrm{~dB}$ and, the return loss is greater than $20 \mathrm{~dB}$.

\section{MODELING OF THE CIRCULAR LOADED DGS STRUCTURE}

In the previous Section, the circular DGS structure is modeled by employing a single resonant parallel RLC circuit model, as depicted in Figure 5. Hence, In configuration two, the EM simulations show two transmission zeros, therefore the equivalent circuit of the loaded DGS cell has to be modelled using two cascaded parallel resonance circuits may be represented in Figure 9. 


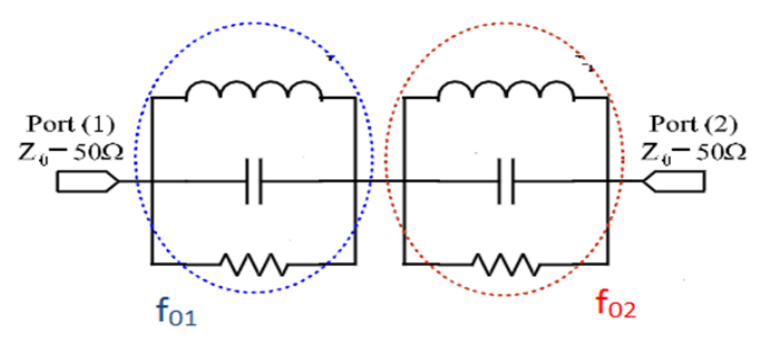

Figure 9. RLC-equivalent circuit for CPW DGS loaded filter

The equivalent impedance of the cascaded parallel resonant circuits can be expressed as:

$$
Z=\frac{1}{\frac{1}{\mathrm{R}_{1}}+\frac{1}{j w L_{1}}+\frac{1}{j w C_{1}}}+\frac{1}{\frac{1}{R_{2}}+\frac{1}{j w L_{2}}+\frac{1}{j w C_{2}}}
$$

where the subscripts ' 1 ' and ' 2 ' denote the two different resonant circuits. The reflection coefficient, S11 is defined as:

$$
S_{11}=\frac{Z_{0}}{2 Z_{0}+Z}=\frac{\left(\frac{j w L_{1}}{1-w^{2} L_{1} C_{1}}+\frac{j w L_{2}}{1-w^{2} L_{2} C_{2}}\right)}{2 Z_{0}+\left(\frac{j w L_{1}}{1-w^{2} L_{1} C_{1}}+\frac{j w L_{2}}{1-w^{2} L_{2} C_{2}}\right)}
$$

The above expression has two poles at the maximum value of S11 by differentiating it with respect to the angular frequency ' $w$ ':

$$
W_{01}=\frac{1}{\sqrt{L_{1} C_{1}}} \quad \text { and } \quad W_{02}=\frac{1}{\sqrt{L_{2} C_{2}}}
$$

The transmission coefficient is given by:

$$
S_{21}=\frac{2 Z_{0}}{2 Z_{0}+Z}=\frac{2 Z_{0}}{2 Z_{0}+\left(\frac{j w L_{1}}{1-w^{2} L_{1} C_{1}}+\frac{j w L_{2}}{1-w^{2} L_{2} C_{2}}\right)}
$$

The full transmission frequency is given by:

$$
W_{p}=\frac{\sqrt{C_{1} w_{1}^{2}+C_{2} w_{2}^{2}}}{\sqrt{C_{1}+C_{2}}}
$$

The $3 \mathrm{~dB}$ cut-off angular frequency, $\mathrm{w}_{\mathrm{c}}$, can be determined as :

$$
\left|\mathrm{S}_{21}\right|_{\mathrm{w}=\mathrm{w}_{\mathrm{c}}}=\frac{1}{\sqrt{2}} \quad \text { gives } \quad\left|1+\frac{\frac{j \mathrm{wL}_{1}}{1-\mathrm{w}^{2} \mathrm{~L}_{1} \mathrm{C}_{1}}+\frac{j \mathrm{wL}_{2}}{1-\mathrm{w}^{2} \mathrm{~L}_{2} \mathrm{C}_{2}}}{2 \mathrm{Z}_{0}}\right|=\sqrt{2}
$$

From (6), (8) and (9), the equivalent circuit parameters can be extracted, whereby

$$
\begin{aligned}
& \mathrm{C}_{1}=\frac{\mathrm{w}_{\mathrm{c}}}{2 \mathrm{Z}_{0}}\left(\frac{\left(w_{p}^{2}-w_{c}^{2}\right)\left(w_{02}^{2}-w_{01}^{2}\right)}{\left(w_{01}^{2}-w_{c}^{2}\right)\left(w_{02}^{2}-w_{c}^{2}\right)\left(w_{p}^{2}-w_{01}^{2}\right)}\right. \\
& \mathrm{C}_{2}=\frac{\mathrm{w}_{\mathrm{c}}}{2 \mathrm{Z}_{0}}\left(\frac{\left(w_{p}^{2}-w_{c}^{2}\right)\left(w_{02}^{2}-w_{01}^{2}\right)}{\left(w_{01}^{2}-w_{c}^{2}\right)\left(w_{02}^{2}-w_{c}^{2}\right)\left(w_{02}^{2}-w_{p}^{2}\right)}\right.
\end{aligned}
$$

The inductances L1 and L2 values can be obtained from (12) as:

$$
L_{1}=\frac{1}{w_{01}^{2} C_{1}} \quad, \quad L_{2}=\frac{1}{w_{02}^{2} C_{2}}
$$


The resistances R1 and R2 of the circuit model are determined around the resonant frequencies $\omega 01$ and $\omega 02$, respectively. At the first resonant frequency $\omega 01$, the equivalent impedance is $\mathrm{ZS} 1 \approx \mathrm{R} 1$ and the transmission loss $\mathrm{S} 21$ is given by:

$$
\begin{aligned}
& \left|S_{21}\right|_{\mathrm{w}=\mathrm{w}_{01}}=\left|\frac{2 \mathrm{Z}_{0}}{2 \mathrm{Z}_{0}+\mathrm{Z}_{\mathrm{s} 1}}\right|=\frac{2 \mathrm{Z}_{0}}{2 \mathrm{Z}_{0}+\mathrm{R}_{1}} \quad \text { gives: } \\
& \mathrm{R}_{1}=2 \mathrm{Z}_{0} \frac{1-\left|\mathrm{S}_{21}\right|_{\mathrm{w}_{01}}}{\left|\mathrm{~S}_{21}\right|_{\mathrm{w}_{01}}}
\end{aligned}
$$

At the second resonant frequency $\omega_{02}$, the equivalent impedance is $\mathrm{ZS} 2 \approx \mathrm{R} 2$ and the transmission loss $\mathrm{S} 21$ is given by:

$$
\begin{aligned}
& \left|S_{21}\right|_{\mathrm{w}=\mathrm{w}_{02}}=\left|\frac{2 \mathrm{Z}_{0}}{2 \mathrm{Z}_{0}+\mathrm{Z}_{\mathrm{s} 2}}\right|=\frac{2 \mathrm{Z}_{0}}{2 \mathrm{Z}_{0}+\mathrm{R}_{2}} \text { gives : } \\
& \mathrm{R}_{2}=2 \mathrm{Z}_{0} \frac{1-\left|\mathrm{S}_{21}\right|_{\mathrm{w}_{02}}}{\left|\mathrm{~S}_{21}\right|_{\mathrm{w}_{02}}}
\end{aligned}
$$

In order to show the validity of the equivalent circuit and the extracted parameters for the proposed unit DGS loaded structure as in the previous two examples, both equivalent circuit and EM simulation S-parameters are shown in Figure 10, using complete structure with proposed modified version.

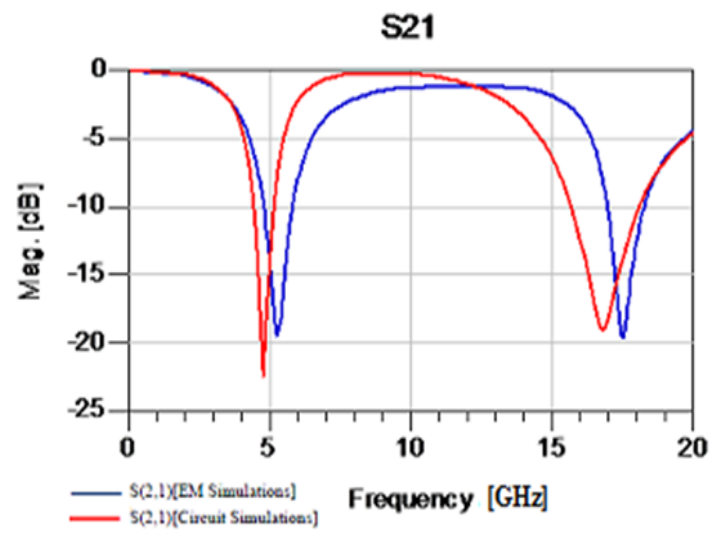

Figure 10. Comparison on parameter extraction of equivalent circuit and EM simulation results for the DGS loaded structure

As results shown, the proposed equivalent circuit results obviously provided good agreement with the EM simulation results. Using complete equivalent circuit and proposed modified version. The values of the equivalent circuit parameters for the cascaded parallel resonance circuits may be represented in Table 2 .

Table 2. Parameters of proposed RLC-equivalent circuit of DGS LOADED

\begin{tabular}{cc}
\hline 1stTransmission Zero & 2nd Transmission Zero \\
\hline $\mathrm{R} 1=1229.8 \Omega$ & $\mathrm{R} 2=790.3714 \Omega$ \\
$\mathrm{C} 1=0.8137 \mathrm{pF}$ & $\mathrm{C} 2=0.38628 \mathrm{pF}$ \\
$\mathrm{L} 1=1.352 \mathrm{nH}$ & $\mathrm{L} 2=0.23248 \mathrm{nH}$ \\
\hline
\end{tabular}

\section{DISTRIBUTION OF SURFACE CURRENT AT PASSBAND AND STOPBAND FREQUENCY \\ RANGES}

The aim of this investigation is to demonstrate the relationship between the EM-simulation results and the surface current density distribution of the proposed O-DGS stopband filter, basing on behaviors of surface current density energies. Figure 11 shows the current distribution simulated at two different frequencies respectively in the passband Figure 11(a) and stopband Figure 11(b). 


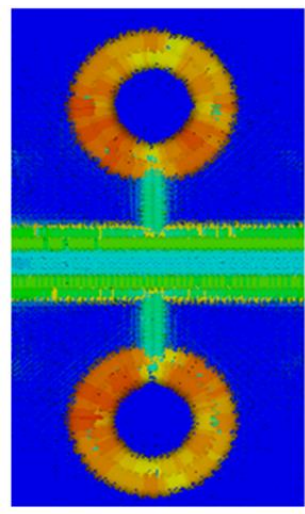

(a)

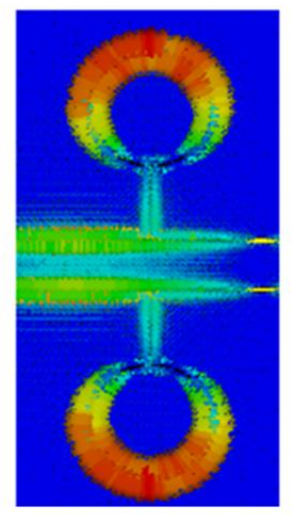

(b)

Figure 11. Simulated surface current density. (a) at $\mathrm{f}=10 \mathrm{GHz}$, (b) at $\mathrm{f}=5.5 \mathrm{GHz}$

As Figure 11(a) shows, we can observe that the current energies is important along the proposed filter structure specially at the O-DGS in the passband region at the frequency of $10 \mathrm{GHz}$, which means that radio frequency magnetic energy was transmitted from the input to the output. In the other side, Figure 11(b), shows the current energies in the stopband region at the frequency of $5.5 \mathrm{GHz}$. At this frequency, there is a high current distribution in the first part of structure around the port 1 and the O-DGS unit and no current near to the output port 2, therefore no a transmission of RF power from the input port to the output port, which indicated that the structure is in the stopband state. This experiment proves that proposed circular DGS loaded filter has similar behaviors between the EM-simulation results and the energies distribution, both have good performances.

\section{CONCLUSION}

In this article, a new compact coplanar stopband filter using circular loaded with O-DGS cell, with excellent suppressed, sharp rejection slope and wide passband is presented and validated in simulation. An RLC equivalent circuit proposed and validated in simulation, all have good performances compared with the energies distribution behaviors. The developed filter can be a good candidate for rejects WLAN frequency at around $5 \mathrm{GHz}$. The circular DGS loaded introduced in the CPW line permits to achieve a multiband stop band filter with good performances. The originality of this work is that the final circuit is a miniature using an optimization method for generating multi-band characteristics without using complicated topology.

\section{ACKNOWLEDGEMENTS}

We thank Mr. Mhamed Latrach from ESEO Angers in France, for allowing us to use and to perform simulations by using electromagnetic solvers and measurement instruments.

\section{REFERENCES}

[1] H. J. Chen, et al., "A novel cross-shape DGS applied to design ultra-wide stop-band low-pass filters," IEEE Microwave and Wireless Components Letters, vol. 16, pp. 252-254, 2006.

[2] J. S. Lim, et al., "A spiral-shaped defected ground structure for coplanar waveguide," IEEE Microwave and Wireless Components Letters, vol. 12, pp. 330-332, 2002.

[3] J. J. Koo, et al., "A new DGS unequal power divider,” European Microwave Conference, pp. 556-559, 2007.

[4] J. P. Thakur and P. Jun-Seok, "A new design approach for circular polarize antenna with DGS under the unbalanced feed-lines," 36th European Microwave Conference, pp. 1483-1485, 2006.

[5] L. H. Weng, et al., "An overview on defencted ground structure," Progress in Electromag- netics Research B, vol. 7, pp. 173-189, 2008.

[6] V. Radisic, et al., "A novel 2-D photonic bandgap Structure for microstrip lines," IEEE Trans. Microwave and Guided Wave Lett., vol. 8, pp. 69-71, 1998.

[7] A. B. Abdel-Rahman, et al., "Control of bandstop response of hi-lo microstrip low-pass filter using slot in the ground plane," IEEE Trans. Microw. Theory Tech., vol. 52, pp. 1008-1013, 2004.

[8] M. K. Mandal and S. Sanyal, "A novel defected ground structure for planar circuits," IEEE Microwave and Wireless Components Letters, vol. 16, pp. 93-95, 2006. 
[9] E. Sghir, et al., "A Novel Compact CPW LowPass Filter Integrating Periodic Triangle DGS Cells," Transactions on Machine Learning and Artificial Intelligence, vol. 5, pp. 457-462, 2017.

[10] D. Guha, et al., "Microstrip and Printed Antennas: New Trends, Techniques and Application," John Wiley\& Sons, pp. 387-434, 2010.

[11] D. Ahn, et al., "A design of the low-pass filter using the novel microstrip defected ground structure," IEEE Trans. Microw. Theory Tech., vol. 49, pp. 86-91, 2001.

[12] M. K. Khandelwal,et al., "Defected Ground Structure: Fundamentals, Analysis, and Applications in Modern Wireless Trends," International Journal of Antennas and Propagation, 2017.

[13] I. Chang and B. Lee, "Design of defected ground structures for harmonic control of active microstrip antenna," Proceedings of the IEEE Antennas and Propagation Society International Symposium, IEEE, San Antonio, Tex, USA, vol. 2, pp. 852-855, 2002.

[14] D. Ahn, et al., "A design of the low-pass filter using the novel microstrip defected ground structure," IEEE Trans. Microw. Theory Tech., vol. 49, pp. 86-93, 2001. 